\title{
La heparina no fraccionada subcutánea sin control de laboratorio sería una alternativa de tratamiento para el tromoembolismo venoso
}

\author{
Unfractionated heparin without laboratory as a treatment alternative for venous thromboembolism.
}

Kearon C y col. JAMA 2006; 296:935-42.

\section{Objetivo}

Determinar si la heparina no fraccionada (HNF) subcutánea en una dosis fija ajustada al peso es tan efectiva y segura como la de bajo peso molecular (HBPM) para el tratamiento inicial del tromboembolismo venoso (TEV).

\section{Diseño}

Ensayo clínico aleatorizado y controlado, con intervención abierta* y evaluación de eventos ciega.

\section{Lugar}

Seis centros universitarios de Canadá y Nueva Zelanda.

\section{Pacientes}

708 adultos con tromboembolismo de pulmón (TEP) sintomático y trombosis venosa profunda (TVP) de miembros inferiores sintomática o asintomática.

\section{Intervención}

En el grupo intervención se administró a los pacientes HNF subcutánea, la primera dosis a razón de $333 \mathrm{U} / \mathrm{Kg}$ seguida de una dosis fija de $250 \mathrm{U} / \mathrm{Kg}$ cada $12 \mathrm{~h}$ sin ajustar al KPTT (tiempo de tromboplastina parcial activado con caolín). El grupo control recibió $100 \mathrm{UI} / \mathrm{Kg}$ cada $12 \mathrm{~h}$ de HBPM (dalteparina o enoxaparina). Desde el primer día ambos grupos fueron tratados con warfarina, que quedó luego como único tratamiento hasta completar tres meses.

\section{Medición de resultados principales}

Recurrencia del TEV durante los primeros tres meses y hemorragia mayor durante los primeros diez días. Dada la baja tasa de reclutamiento el estudio debió ser suspendido sin llegar al número planeado inicialmente, de 824 pacientes.

\section{Resultados principales}

El tratamiento fue ambulatorio en el $72 \%$ de los pacientes del grupo HNF y en $68 \%$ del grupo control. Ver tabla 1.

Tabla 1: resultados principales.

\begin{tabular}{|c|c|c|c|}
\hline $\begin{array}{l}\text { Pacientes } y \\
\text { resultados }\end{array}$ & $\begin{array}{c}\text { Heparina no } \\
\text { fraccionada ( } n=355)\end{array}$ & $\begin{array}{l}\text { Heparina de bajo peso } \\
\text { molecular ( } \mathrm{n}=353)\end{array}$ & RRA $^{2}$ (IC $95 \%$ ) \\
\hline Recurrencia de TEV & $13(3,8 \%)$ & $12(3,4 \%)$ & $0,4(-2,6$ a 3,3$)$ \\
\hline Sangrado mayor & $4(1,1 \%)$ & $5(1,4 \%)$ & $-0,3 \quad(-2,3$ a 1,7$)$ \\
\hline Muerte & $18(5,2 \%)$ & $22(6,3 \%)$ & $-1,1 \quad(-4,6$ a 2,5$)$ \\
\hline
\end{tabular}

RRA a: reducción de riesgo absoluto.

\section{Conclusiones}

La HNF subcutánea es tan segura y efectiva como la HBPM en el tratamiento del TEV.

Palabras claves:Ensayo clínico, heparina no fraccionada, heparina de bajo peso molecular, tromboembolismo venoso, tratamiento ambulatorio Fuente de financiamiento:Fundación canadiense del corazón y el ACV

\section{Comentario}

La HNF endovenosa ajustada según el KPTT fue el primer tratamiento en mostrar reducción de la mortalidad del TEP ${ }^{1}$. Con el tiempo, las HBPM demostraron ser tan efectivas y seguras. A pesar de su mayor costo, pasaron a ser el tratamiento de primera elección por no requerir controles de KPTT y por la posibilidad de tratamiento ambulatorio y la consiguiente reducción de costos globales ${ }^{2}$. Por eso resulta paradójico que la medicación pionera para tratar esta enfermedad necesite ahora compararse con el actual tratamiento de referencia: las HBPM.

El grupo de Kearon -no financiado por la industria farmacéutica- ya había publicado en el 2000 un trabajo usando HNF subcutánea cada $12 \mathrm{~h}$ y ajustando la dosis al $\mathrm{KPTT}^{3}$. En el estudio actual demostró que tampoco se requieren controles de laboratorio. Las limitaciones principales de este trabajo están dadas por su diseño abierto* (que se compensó con la evaluación ciega de eventos) el menor número de pacientes respecto al planeado (si bien el poder fue suficiente para detectar una diferencia clínicamente relevante) y por el número significativo de pacientes excluidos luego de la aleatorización (diez en un grupo y uno en el otro) que no se tuvieron en cuenta para el análisis de eficacia. Si bien no fue diseñado para tal efecto, el hecho de que un $72 \%$ de los pacientes fuera tratados en domicilio hace que el tratamiento ambulatorio parezca una opción a tener en cuenta.

\section{Conclusiones del comentador}

El resultado de este estudio es muy prometedor para países poco desarrollados como los nuestros. Sin embargo, parece algo prematuro todavía recomendar el cambio del tratamiento del TEV en la práctica clínica de todos los días.

Daniel Roubicek [ Especialista en Clínica Médica. Instituto Médico Bolívar. Hospital Privado de Comunidad. Mar del Plata. ]

Roubicek D. La heparina no fraccionada subcutánea sin control de laboratorio sería una alternativa de tratamiento para el tromoembolismo venoso. Evid. actual. práct. ambul; 10(2): 41, mar-abr.2007. Comentado de: Kearon C y col. Fixed-Dose Heparin (FIDO) Investigators. Comparison of fixeddose weight-adjusted unfractionated heparin and low-molecular-weight heparin for acute treatment of venous thromboembolism. JAMA. 2006; 296(8):935-42. PMID: 16926353.

\section{Referencias}

1. Carson JL. Subcutaneous unfractionated heparin vs low-molecular-weight heparin for acute thromboembolic disease. Issues of efficacy and cost. JAMA 2006;296:991-3. 2. Hull RD, Raskob GE, Pineo GF y col. Subcutaneous low-molecular-weight heparin vs warfarin for prophylaxis of deep vein thrombosis after hip or knee implantation. An economic perspective. Arch Intern Med 1997;157:298-303

3. Kearon C, Harrison L, Crowther M y col. Optimal dosing of subcutaneous unfractionated heparin for the treatment of deep vein thrombosis. Tromb Res 2000;97:395-403. 